\title{
Asymptotic behaviour of solution for multidimensional viscoelasticity equation with nonlinear source term
}

\author{
Runzhang $\mathrm{Xu}^{1 *}$, Jie Liu', Yi Niu² and Shaohua Chen ${ }^{3}$
}

\section{"Correspondence:}

xurunzh@yahoo.com.cn

${ }^{1}$ College of Science, Harbin

Engineering University, Harbin,

150001, People's Republic of China

Full list of author information is

available at the end of the article

\begin{abstract}
In this paper we study the initial-boundary value problem of the multidimensional viscoelasticity equation with nonlinear source term $u_{t t}-\Delta u_{t}-\sum_{i=1}^{N} \frac{\partial}{\partial x_{i}} \sigma_{i}\left(u_{x_{j}}\right)=f(u)$. By using the potential well method, we first prove the global existence. Then we prove that when time $t \rightarrow+\infty$, the solution decays to zero exponentially under some assumptions on nonlinear functions and the initial data.
\end{abstract}

\section{Introduction}

This paper considers the initial-boundary value problem (IBVP) of the multidimensional viscoelasticity equation with nonlinear source term

$$
\begin{aligned}
& u_{t t}-\Delta u_{t}-\sum_{i=1}^{N} \frac{\partial}{\partial x_{i}} \sigma_{i}\left(u_{x_{i}}\right)=f(u), \quad x \in \Omega, t>0, \\
& u(x, 0)=u_{0}(x), \quad u_{t}(x, 0)=u_{1}(x), \quad x \in \Omega, \\
& u(x, t)=0, \quad x \in \partial \Omega, t \geq 0,
\end{aligned}
$$

where $u(x, t)$ is the unknown function with respect to the spacial variable $x \in \Omega$ and the time variable $t, \Omega \subset \mathbb{R}^{N}$ is a bounded domain.

The viscoelasticity equation

$$
u_{t t}-u_{x x t}=\sigma\left(u_{x}\right)_{x}
$$

was suggested and studied by Greenberg et al. [1,2] from viscoelasticity mechanics in 1968. Under the condition $\sigma^{\prime}(s)>0$ and higher smooth conditions on $\sigma(s)$ and the initial data, they obtained the global existence of classical solutions for the initial-boundary value problem of Eq. (1.4).

After that many authors [3-11] studied the global well-posedness of IBVP for Eq. (1.4). In [3-10] the global existence, uniqueness and stability of solution were studied thoroughly. And in [11] the blow up of solution was discussed. Furthermore, in [12-14] the global existence of solution for IBVP of some multidimensional viscoelasticity equation was considered. And in [11] the blow up of solution for IBVP of the multidimensional generalisation

\section{Springer}

(c) 2013 Xu et al.; licensee Springer. This is an Open Access article distributed under the terms of the Creative Commons Attribution License (http://creativecommons.org/licenses/by/2.0), which permits unrestricted use, distribution, and reproduction in any medium, provided the original work is properly cited. 
of Eq. (1.4) was proved. Recently, in [15] and [16], the IBVP of the multidimensional viscoelasticity equation with nonlinear damping and source terms

$$
\begin{aligned}
& u_{t t}-\Delta u_{t}-\sum_{i=1}^{N} \frac{\partial}{\partial x_{i}} \sigma_{i}\left(u_{x_{i}}\right)+f\left(u_{t}\right)=g(u), \quad x \in \Omega, t>0, \\
& u(x, 0)=u_{0}(x), \quad u_{t}(x, 0)=u_{1}(x), \quad x \in \Omega, \\
& u(x, t)=0, \quad x \in \partial \Omega, t \geq 0,
\end{aligned}
$$

was studied, and by using the potential well method, the global existence of weak solution was proved under some assumptions on nonlinear functions $\sigma_{i}(s), f(s), g(s)$ and the initial data. But we do not know how the global solution behaves as the time goes to infinity, namely the asymptotic behaviour of problem (1.1)-(1.3) is still open up to now. In the present paper, we try to study this problem by the multiplier method [17-22].

The main purpose of present paper is to consider the asymptotic behaviour of solution for problem (1.1)-(1.3). Since in the proof of the asymptotic behaviour of solution the global existence theory is required, it is necessary to give the proof of global existence of solution for problem (1.1)-(1.3).

In this paper, suppose that $\sigma(s)=\left(\sigma_{1}(s), \ldots, \sigma_{N}(s)\right)$ and $f(s)$ satisfy the following assumptions:

$$
\begin{gathered}
\left(\mathrm{H}_{1}\right)\left\{\begin{array}{c}
\text { (i) } \sigma \in C^{1}, \sigma_{i}(0)=0, \min _{1 \leq i \leq N}\left\{\inf _{s \in \mathbb{R}} \sigma_{i}(s)\right\}=a>0 ; \\
\text { (ii) } \sigma_{i}(s) s \geq A|s|^{m+1},\left|\sigma_{i}(s)\right| \leq B\left(|s|^{m}+1\right), \\
\text { where } A \text { and } B \text { are both positive constants; } \\
\text { (iii) }(l+1) G_{i}(s) \geq s \sigma_{i}(s), \text { where } G_{i}(s)=\int_{0}^{s} \sigma_{i}(\tau) \mathrm{d} \tau .
\end{array}\right. \\
\left(\mathrm{H}_{2}\right)\left\{\begin{array}{l}
\left(\text { i) } f \in C,|f(u)| \leq b|u|^{q}, \forall u \in \mathbb{R} ;\right. \\
\text { (ii) }(p+1) F(u) \leq u f(u) \leq(r+1) F(u), \forall u \in \mathbb{R},
\end{array}\right.
\end{gathered}
$$

where the constants in $\left(\mathrm{H}_{1}\right)$ and $\left(\mathrm{H}_{2}\right)$ are all positive and satisfy

$$
\begin{aligned}
& 2 \leq m+1<q+1 \leq \frac{N(m+1)}{N-m-1} \text { for } m+1<N, \\
& 2 \leq m+1<q+1<\infty \text { for } m+1 \geq N, \\
& 1 \leq l<p \leq r .
\end{aligned}
$$

In this paper, we first give some definitions and lemmas (Section 2). Then we prove the global existence of solution (Section 3). Finally, we prove the asymptotic behaviour of solution (Section 4).

In this paper, we denote $\|\cdot\|_{L^{p}(\Omega)}$ by $\|\cdot\|_{p},\|\cdot\|=\|\cdot\|_{L^{2}(\Omega)}$ and $(u, v)=\int_{\Omega} u v \mathrm{~d} x$.

\section{Preliminaries}

In this section, we will give some definitions and prove some lemmas for problem (1.1)(1.3). 
For problem (1.1)-(1.3), we define

$$
\begin{aligned}
& J(u)=\sum_{i=1}^{N} \int_{\Omega} G_{i}\left(u_{x_{i}}\right) \mathrm{d} x-\int_{\Omega} F(u) \mathrm{d} x, \\
& F(u)=\int_{0}^{u} f(s) \mathrm{d} s, G_{i}(s)=\int_{0}^{s} \sigma_{i}(\tau) \mathrm{d} \tau, \quad 1 \leq i \leq N, \\
& I(u)=\sum_{i=1}^{N} \int_{\Omega} u_{x_{i}} \sigma_{i}\left(u_{x_{i}}\right) \mathrm{d} x-\int_{\Omega} u f(u) \mathrm{d} x, \\
& I_{\delta}(u)=\delta \sum_{i=1}^{N} \int_{\Omega} u_{x_{i}} \sigma_{i}\left(u_{x_{i}}\right) \mathrm{d} x-\int_{\Omega} u f(u) \mathrm{d} x, \quad \delta>0, \\
& d=\inf _{u \in \mathcal{N}} J(u), \quad \mathcal{N}=\left\{u \in W_{0}^{1, m+1}(\Omega) \mid I(u)=0 u \neq 0\right\}, \\
& E(t)=\frac{1}{2}\left\|u_{t}\right\|^{2}+\sum_{i=1}^{N} \int_{\Omega} G_{i}\left(u_{x_{i}}\right) \mathrm{d} x-\int_{\Omega} F(u) \mathrm{d} x=\frac{1}{2}\left\|u_{t}\right\|^{2}+J(u) .
\end{aligned}
$$

Remark 2.1 Note that the definitions of $J(u)$ and $I(u)$ in the present paper are different from those in [11] and [15]. The definitions given in this paper will be shown more natural and rational because they are a part of the total energy $E(t)$.

Lemma 2.2 Let $\left(\mathrm{H}_{1}\right)$ and $\left(\mathrm{H}_{2}\right)$ hold. Set

$$
\bar{\sigma}_{i}(s)=\sigma_{i}(s)-a s, \quad \bar{G}_{i}(s)=\int_{0}^{s} \bar{\sigma}_{i}(s) \mathrm{d} \tau .
$$

Then the following hold:

(i) $\bar{\sigma}_{i}(s)$ is increasing and $s \bar{\sigma}_{i}(s) \geq 0 \forall s \in \mathbb{R}$;

(ii) $0 \leq \bar{G}_{i}(s) \leq s \bar{\sigma}_{i}(s) \forall s \in \mathbb{R}$.

Proof This lemma follows from $\bar{\sigma}_{i}(0)=0$ and $\bar{\sigma}_{i}^{\prime}(s) \geq 0$.

Lemma 2.3 Let $\left(\mathrm{H}_{1}\right)$ and $\left(\mathrm{H}_{2}\right)$ hold, $u \in W_{0}^{1, m+1}(\Omega)$. Then the following hold:

(i) If $0<\|\nabla u\|_{m+1}<r(\delta)$, then $I_{\delta}(u)>0$;

(ii) If $I_{\delta}(u)<0$, then $\|\nabla u\|_{m+1}>r(\delta)$;

(iii) If $I_{\delta}(u)=0$, then $\|\nabla u\|_{m+1} \geq r(\delta)$,

where

$$
r(\delta)=\left(\frac{A \delta}{b C_{*}^{q+1}}\right)^{\frac{1}{q-m}}, \quad C_{*}=\sup _{u \in W_{0}^{1, m+1}(\Omega) / 0} \frac{\|u\|_{q+1}}{\|\nabla u\|_{m+1}} .
$$

Proof

(i) If $0<\|\nabla u\|_{m+1}<r(\delta)$, then we have

$$
\begin{aligned}
\int_{\Omega} u f(u) \mathrm{d} x & \leq \int_{\Omega}|u f(u)| \mathrm{d} x \leq b \int_{\Omega}|u|^{q+1} \mathrm{~d} x \\
& =b\|u\|_{q+1}^{q+1} \leq b C_{*}^{q+1}\|\nabla u\|_{m+1}^{q+1}
\end{aligned}
$$




$$
\begin{aligned}
& =\frac{b C_{*}^{q+1}}{A}\|\nabla u\|_{m+1}^{q-m} A\|\nabla u\|_{m+1}^{m+1} \\
& <\delta \sum_{i=1}^{N} \int_{\Omega} u_{x_{i}} \sigma_{i}\left(u_{x_{i}}\right) \mathrm{d} x
\end{aligned}
$$

which gives $I_{\delta}(u)>0$.

(ii) If $I_{\delta}(u)<0$, then we have

$$
\delta A\|\nabla u\|_{m+1}^{m+1} \leq \delta \sum_{i=1}^{N} \int_{\Omega} u_{x_{i}} \sigma_{i}\left(u_{x_{i}}\right) \mathrm{d} x<\int_{\Omega} u f(u) \mathrm{d} x \leq b C_{*}^{q+1}\|\nabla u\|_{q-m}^{m+1}\|\nabla u\|_{m+1}^{m+1},
$$

which gives

$$
\|\nabla u\|_{m+1}>r(\delta)
$$

(iii) If $I_{\delta}(u)=0$ and $u \neq 0$, then by

$$
\delta A\|\nabla u\|_{m+1}^{m+1} \leq \delta \sum_{i=1}^{N} \int_{\Omega} u_{x_{i}} \sigma_{i}\left(u_{x_{i}}\right) \mathrm{d} x<\int_{\Omega} u f(u) \mathrm{d} x \leq b C_{*}^{q+1}\|\nabla u\|_{m+1}^{q-m}\|\nabla u\|_{m+1}^{m+1}
$$

we get

$$
\|\nabla u\|_{m+1} \geq r(\delta)
$$

Lemma 2.4 Let $\left(\mathrm{H}_{1}\right)$ and $\left(\mathrm{H}_{2}\right)$ hold. Then the following holds:

$$
d \geq d_{0}=\frac{(p-l) A}{(p+1)(l+1)}\left(\frac{A}{b C_{*}^{q+1}}\right)^{\frac{m+1}{q-m}}
$$

Proof For any $u \in \mathcal{N}$, by Lemma 2.3, we have $\|\nabla u\|_{m+1} \geq r(1)$ and

$$
\begin{aligned}
J(u) & =\sum_{i=1}^{N} \int_{\Omega} G_{i}\left(u_{x_{i}}\right) \mathrm{d} x-\int_{\Omega} F(u) \mathrm{d} x \\
& \geq \frac{1}{l+1} \sum_{i=1}^{N} \int_{\Omega} u_{x_{i}} \sigma_{i}\left(u_{x_{i}}\right) \mathrm{d} x-\frac{1}{p+1} \int_{\Omega} u f(u) \mathrm{d} x \\
& =\left(\frac{1}{l+1}-\frac{1}{p+1}\right) \sum_{i=1}^{N} \int_{\Omega} u_{x_{i}} \sigma_{i}\left(u_{x_{i}}\right) \mathrm{d} x+\frac{1}{p+1} I(u) \\
& =\frac{p-l}{(p+1)(l+1)} \sum_{i=1}^{N} \int_{\Omega} u_{x_{i}} \sigma_{i}\left(u_{x_{i}}\right) \mathrm{d} x \\
& \geq \frac{p-l}{(p+1)(l+1)} A\|\nabla u\|_{m+1}^{m+1} \\
& \geq \frac{p-l}{(p+1)(l+1)} A r^{m+1}(1)=\frac{(p-l) A}{(p+1)(l+1)}\left(\frac{A}{b C_{*}^{q+1}}\right)^{\frac{m+1}{q-m}},
\end{aligned}
$$

which gives (2.1). 
Now, for problem (1.1)-(1.3), we define

$$
W=\left\{u \in W_{0}^{1, m+1}(\Omega) \mid I(u)>0\right\} \cup\{0\} .
$$

\section{Global existence of solution}

In this section, we prove the global existence of weak solution for problem (1.1)-(1.3).

Definition 3.1 We call $u=u(x, t)$ a weak solution of problem (1.1)-(1.3) on $\Omega \times[0, T)$ if $u \in L^{\infty}\left(0, T ; W_{0}^{1, m+1}(\Omega)\right), u_{t} \in L^{\infty}\left(0, T ; L^{2}(\Omega)\right) \cap L^{2}\left(0, T ; H_{0}^{1}(\Omega)\right)$ satisfying

(i)

$$
\begin{aligned}
& \left(u_{t}, v\right)+(\nabla u, \nabla v)+\sum_{i=1}^{N} \int_{0}^{t}\left(\sigma_{i}\left(u_{x_{i}}\right), v_{x_{i}}\right) \mathrm{d} \tau \\
& \quad=\int_{0}^{t}(f(u), v) \mathrm{d} \tau+\left(u_{1}, v\right)+\left(\nabla u_{0}, \nabla v\right), \quad \forall v \in W_{0}^{1, m+1}(\Omega), \forall t \in[0, t),
\end{aligned}
$$

(ii)

$$
u(x, 0)=u_{0}(x) \quad \text { in } W_{0}^{1, m+1}(\Omega) ; \quad u_{t}(x, 0)=u_{1}(x) \quad \text { in } L^{2}(\Omega)
$$

Theorem 3.2 Let $\left(\mathrm{H}_{1}\right)$ and $\left(\mathrm{H}_{2}\right)$ hold, $u_{0}(x) \in W_{0}^{1, m+1}(\Omega), u_{1}(x) \in L^{2}(\Omega)$. Assume that $E(0)<d, u_{0}(x) \in W$. Then problem (1.1)-(1.3) admits a global weak solution $u \in L^{\infty}(0, \infty$; $\left.W_{0}^{1, m+1}(\Omega)\right)$ and $u_{t} \in L^{\infty}\left(0, \infty ; L^{2}(\Omega)\right) \cap L^{2}\left(0, \infty ; H_{0}^{1}(\Omega)\right)$.

Proof Let $\left\{w_{j}(x)\right\}_{j=1}^{\infty}$ be a system of base functions in $W_{0}^{1, m+1}(\Omega)$. Construct the approximate solutions of problem (1.1)-(1.3)

$$
u_{n}(x, t)=\sum_{j=1}^{n} g_{j n}(t) w_{j}(x), \quad n=1,2, \ldots
$$

satisfying

$$
\begin{aligned}
& \left(u_{n t t}, w_{s}\right)+\left(\nabla u_{n t}, \nabla w_{s}\right)+\sum_{i=1}^{N}\left(\sigma_{i}\left(u_{n x_{i}}\right), w_{s x_{i}}\right)=\left(f\left(u_{n}\right), w_{s}\right), \quad s=1,2, \ldots, N, \\
& u_{n}(x, 0)=\sum_{j=1}^{n} g_{j n}(0) w_{j}(x) \rightarrow u_{0}(x) \quad \text { in } W_{0}^{1, m+1}(\Omega), \\
& u_{n t}(x, 0)=\sum_{j=1}^{n} g_{j n}^{\prime}(0) w_{j}(x) \rightarrow u_{1}(x) \quad \text { in } L^{2}(\Omega) .
\end{aligned}
$$

Multiplying (3.1) by $g_{s n}^{\prime}(t)$ and summing for $s$, we get

$$
\frac{\mathrm{d}}{\mathrm{d} t} E_{n}(t)+\left\|\nabla u_{n t}\right\|^{2}=0, \quad 0 \leq t<\infty
$$

and

$$
E_{n}(t)+\int_{0}^{t}\left\|\nabla u_{n \tau}\right\|^{2} \mathrm{~d} \tau=E_{n}(0), \quad 0 \leq t<\infty
$$


where

$$
E_{n}(t)=\frac{1}{2}\left\|u_{n t}\right\|^{2}+J\left(u_{n}\right) .
$$

From (3.2) and (3.3), we have $E_{n}(0) \rightarrow E(0)$ as $n \rightarrow \infty$. Hence, for sufficiently large $n$, we have $E_{n}(0)<d$ and

$$
\frac{1}{2}\left\|u_{n t}\right\|^{2}+J\left(u_{n}\right)+\int_{0}^{t}\left\|\nabla u_{n \tau}\right\|^{2} \mathrm{~d} \tau<d, \quad 0 \leq t<\infty
$$

On the other hand, since $W$ is an open set in $W_{0}^{1, m+1}(\Omega)$, Eq. (3.2) implies that for sufficiently large $n$, we have $u_{n}(0) \in W$. Next, we prove that $u_{n}(t) \in W$ for $0<t<\infty$ and sufficiently large $n$. If it is false, then there exists a $t_{0}>0$ such that $u_{n}\left(t_{0}\right) \in \partial W$, i.e. $I\left(u_{n}\left(t_{0}\right)\right)=0$ and $u_{n}\left(t_{0}\right) \neq 0$, i.e. $u_{n}\left(t_{0}\right) \in \mathcal{N}$. So, by the definition of $d$, we get $J\left(u_{n}\left(t_{0}\right)\right) \geq d$, which contradicts (3.6).

From (3.6) we have

$$
\frac{1}{2}\left\|u_{n t}\right\|^{2}+\sum_{i=1}^{N} \int_{\Omega} G_{i}\left(u_{n x_{i}}\right) \mathrm{d} x-\int_{\Omega} F\left(u_{n}\right) \mathrm{d} x+\int_{0}^{t}\left\|\nabla u_{n \tau}\right\|^{2} \mathrm{~d} \tau<d, \quad 0 \leq t<\infty,
$$

which gives

$$
\frac{1}{2}\left\|u_{n t}\right\|^{2}+\frac{1}{l+1} \sum_{i=1}^{N} \int_{\Omega} u_{n x_{i}} \sigma_{i}\left(u_{n x_{i}}\right) \mathrm{d} x-\frac{1}{p+1} \int_{\Omega} u_{n} f\left(u_{n}\right) \mathrm{d} x+\int_{0}^{t}\left\|\nabla u_{n \tau}\right\|^{2} \mathrm{~d} \tau<d
$$

and

$$
\begin{aligned}
& \frac{1}{2}\left\|u_{n t}\right\|^{2}+\frac{p-l}{(p+1)(l+1)} \sum_{i=1}^{N} \int_{\Omega} u_{n x_{i}} \sigma_{i}\left(u_{n x_{i}}\right) \mathrm{d} x+\frac{1}{p+1} I\left(u_{n}\right)+\int_{0}^{t}\left\|\nabla u_{n \tau}\right\|^{2} \mathrm{~d} \tau<d, \\
& 0 \leq t<\infty,
\end{aligned}
$$

which together with $u_{n}(t) \in W$ gives

$$
\frac{1}{2}\left\|u_{n t}\right\|^{2}+\frac{p-l}{(p+1)(l+1)} \sum_{i=1}^{N} \int_{\Omega} u_{n x_{i}} \sigma_{i}\left(u_{n x_{i}}\right) \mathrm{d} x+\int_{0}^{t}\left\|\nabla u_{n \tau}\right\|^{2} \mathrm{~d} \tau<d,
$$

and

$$
\frac{1}{2}\left\|u_{n t}\right\|^{2}+\frac{p-l}{(p+1)(l+1)} A\left\|\nabla u_{n}\right\|_{m+1}^{m+1}+\int_{0}^{t}\left\|\nabla u_{n \tau}\right\|^{2} \mathrm{~d} \tau<d, \quad 0 \leq t<\infty .
$$

From (3.7) we can get

$$
\begin{aligned}
& \left\|\nabla u_{n}\right\|_{m+1}^{m+1}<\frac{(p+1)(l+1)}{p-l} \frac{1}{A} d, \quad 0 \leq t<\infty, \\
& \left\|u_{n t}\right\|^{2}<2 d, \quad 0 \leq t<\infty \\
& \int_{0}^{t}\left\|\nabla u_{n \tau}\right\|^{2} \mathrm{~d} \tau<d, \quad 0 \leq t<\infty
\end{aligned}
$$




$$
\begin{aligned}
& \left\|\sigma_{i}\left(u_{n x_{i}}\right)\right\|_{\frac{m+1}{m}<C,}<\leq t<\infty, \\
& \left\|f\left(u_{n}\right)\right\|_{\frac{q+1}{q}} \leq C, \quad 0 \leq t<\infty .
\end{aligned}
$$

Hence there exist $u, \chi=\left(\chi_{1}, \chi_{2}, \ldots, \chi_{N}\right), \eta$ and a subsequence $\left\{u_{\nu}\right\}$ of $\left\{u_{n}\right\}$ such that as $v \rightarrow \infty, u_{v} \rightarrow u$ in $u \in L^{\infty}\left(0, \infty ; W_{0}^{1, m+1}(\Omega)\right)$ weak-star, and a.e. in $Q=\Omega \times[0, \infty), u_{v t} \rightarrow u_{t}$ in $L^{\infty}\left(0, \infty ; L^{2}(\Omega)\right)$ weak-star and in $L^{2}\left(0, \infty ; H_{0}^{1}(\Omega)\right)$ weakly, $\sigma_{i}\left(u_{v x_{i}}\right) \rightarrow \chi_{i}=\sigma_{i}\left(u_{x_{i}}\right)$ in $L^{\infty}\left(0, \infty ; L^{(m+1)^{\prime}}(\Omega)\right)$ weak-star, $(m+1)^{\prime}=\frac{m+1}{m}, f\left(u_{v}\right) \rightarrow \eta=f(u)$ in $L^{\infty}\left(0, \infty ; L^{(q+1)^{\prime}}(\Omega)\right)$ weak-star, $(q+1)^{\prime}=\frac{q+1}{q}$.

Integrating (3.1) with respect to $t$, we have

$$
\begin{aligned}
& \left(u_{n t}, w_{s}\right)+\left(\nabla u_{n}, \nabla w_{s}\right)+\sum_{i=1}^{N} \int_{0}^{t}\left(\sigma_{i}\left(u_{n x_{i}}\right), w_{s x_{i}}\right) \mathrm{d} \tau \\
& =\int_{0}^{t}\left(f\left(u_{n}\right), w_{s}\right) \mathrm{d} \tau+\left(u_{n t}(0), w_{s}\right)+\left(\nabla u_{n}(0), \nabla w_{s}\right) .
\end{aligned}
$$

Letting $n=v \rightarrow \infty$ in (3.13), we get

$$
\begin{aligned}
& \left(u_{t}, w_{s}\right)+\left(\nabla u, \nabla w_{s}\right)+\sum_{i=1}^{N} \int_{0}^{t}\left(\sigma_{i}\left(u_{x_{i}}\right), w_{s x_{i}}\right) \mathrm{d} \tau \\
& =\int_{0}^{t}\left(f(u), w_{s}\right) \mathrm{d} \tau+\left(u_{1}, w_{s}\right)+\left(\nabla u_{0}, \nabla w_{s}\right), \quad \forall s,
\end{aligned}
$$

and

$$
\begin{aligned}
& \left(u_{t}, v\right)+(\nabla u, \nabla v)+\sum_{i=1}^{N} \int_{0}^{t}\left(\sigma_{i}\left(u_{x_{i}}\right), v_{x_{i}}\right) \mathrm{d} \tau \\
& =\int_{0}^{t}(f(u), v) \mathrm{d} \tau+\left(u_{1}, v\right)+\left(\nabla u_{0}, \nabla v\right), \quad \forall v \in W_{0}^{1, m+1}(\Omega), t>0 .
\end{aligned}
$$

On the other hand, from (3.2) and (3.3), we get $u(x, 0)=u_{0}(x)$ in $W_{0}^{1, m+1}(\Omega), u_{t}(x, 0)=u_{1}(x)$ in $L^{2}(\Omega)$. Therefore $u$ is a global weak solution of problem (1.1)-(1.3).

\section{Asymptotic behaviour of solution}

In this section, we prove the main conclusion of this paper - the asymptotic behaviour of solution for problem (1.1)-(1.3).

Lemma 4.1 Let $\left(\mathrm{H}_{1}\right)$ and $\left(\mathrm{H}_{2}\right)$ hold, $u_{0}(x) \in W_{0}^{1, m+1}(\Omega), u_{1}(x) \in L^{2}(\Omega)$. Then, for the approximate solutions $u_{n}(x, t)$ of problem (1.1)-(1.3) constructed in the proof of Theorem 3.2, the following hold:

(i)

$$
I\left(u_{n}\right)=\left\|u_{n t}\right\|^{2}-\frac{\mathrm{d}}{\mathrm{d} t}\left(\left(u_{n t}, u_{n}\right)+\frac{1}{2}\left\|\nabla u_{n}\right\|^{2}\right)
$$


(ii) Furthermore, if $E(0)<d_{0}$ and $u_{0}(x) \in W$, then for sufficiently large $n$, there exists a $\delta_{1} \in(0,1)$ such that

$$
I\left(u_{n}\right) \geq\left(1-\delta_{1}\right) \sum_{i=1}^{N}\left(\sigma_{i}\left(u_{n x_{i}}\right), u_{n x_{i}}\right)
$$

Proof (i) Multiplying (3.1) by $g_{s n}(t)$ and summing for $s$, we get (4.1).

(ii) From

$$
E(0)<d_{0}=\frac{p-l}{(p+1)(l+1)} A\left(\frac{A}{b C_{*}^{q+1}}\right)^{\frac{m+1}{q-m}}
$$

it follows that there exists a $\delta_{1} \in(0,1)$ such that

$$
E(0)<\frac{p-l}{(p+1)(l+1)} A\left(\frac{A \delta_{1}}{b C_{*}^{q+1}}\right)^{\frac{m+1}{q-m}} \equiv d\left(\delta_{1}\right) .
$$

From (3.2), (3.3) and (4.3), it follows that $E_{m}(0)<d\left(\delta_{1}\right)$ for sufficiently large $n$. Hence from (3.5) we have

$$
\begin{aligned}
& \frac{1}{2}\left\|u_{n t}\right\|^{2}+J\left(u_{n}\right)+\int_{0}^{t}\left\|\nabla u_{n \tau}\right\|^{2} \mathrm{~d} \tau<d\left(\delta_{1}\right), \quad 0 \leq t<\infty, \\
& J\left(u_{n}\right) \leq d\left(\delta_{1}\right),
\end{aligned}
$$

and

$$
\sum_{i=1}^{N} \int_{\Omega} G_{i}\left(u_{n x_{i}}\right) \mathrm{d} x-\int_{\Omega} F\left(u_{n}\right) \mathrm{d} x<d\left(\delta_{1}\right)
$$

which gives

$$
\frac{1}{l+1} \sum_{i=1}^{N} \int_{\Omega} u_{n x_{i}} \sigma_{i}\left(u_{n x_{i}}\right) \mathrm{d} x-\frac{1}{p+1} \int_{\Omega} u_{n} f\left(u_{n}\right) \mathrm{d} x<d\left(\delta_{1}\right)
$$

and

$$
\frac{p-l}{(p+1)(l+1)} \sum_{i=1}^{N} \int_{\Omega} u_{n x_{i}} \sigma_{i}\left(u_{n x_{i}}\right) \mathrm{d} x+\frac{1}{p+1} I\left(u_{n}\right)<d\left(\delta_{1}\right),
$$

which together with $u_{n} \in W$ for sufficiently large $n$ gives

$$
\frac{p-l}{(p+1)(l+1)} A\left\|\nabla u_{n}\right\|_{m+1}^{m+1}<\frac{p-l}{(p+1)(l+1)} A\left(\frac{A \delta_{1}}{b C_{*}^{q+1}}\right)^{\frac{m+1}{q-m}}
$$

and

$$
\left\|\nabla u_{n}\right\|_{m+1}<\left(\frac{A \delta_{1}}{b C_{*}^{q+1}}\right)^{\frac{1}{q-m}}=r\left(\delta_{1}\right)
$$


Hence, by Lemma 2.3, we have $I_{\delta_{1}}\left(u_{n}\right)>0$ or $u_{n}=0$. So, we have

$$
\begin{aligned}
I\left(u_{n}\right) & =\sum_{i=1}^{N} \int_{\Omega} u_{n x_{i}} \sigma_{i}\left(u_{n x_{i}}\right) \mathrm{d} x-\int_{\Omega} u_{n} f\left(u_{n}\right) \mathrm{d} x \\
& =\left(1-\delta_{1}\right) \sum_{i=1}^{N} \int_{\Omega} u_{n x_{i}} \sigma_{i}\left(u_{n x_{i}}\right) \mathrm{d} x+I_{\delta_{1}}\left(u_{n}\right) \geq\left(1-\delta_{1}\right) \sum_{i=1}^{N} \int_{\Omega} u_{n x_{i}} \sigma_{i}\left(u_{n x_{i}}\right) \mathrm{d} x .
\end{aligned}
$$

Theorem 4.2 Let $\left(\mathrm{H}_{1}\right)$ and $\left(\mathrm{H}_{2}\right)$ hold, $u_{0}(x) \in W_{0}^{1, m+1}(\Omega), u_{1}(x) \in L^{2}(\Omega)$. Assume that $E(0)<d_{0}, u_{0}(x) \in W$. Then, for the global weak solution $u$ given in Theorem 3.2, there exist positive constants $C$ and $\lambda$ such that

$$
\left\|u_{t}\right\|^{2}+\|\nabla u\|_{m+1}^{m+1} \leq C e^{-\lambda t}, \quad 0 \leq t<\infty
$$

Proof Let $\left\{u_{n}\right\}$ be the approximate solutions of problem (1.1)-(1.3) in the proof of Theorem 3.2, then (3.4) holds. Multiplying (3.4) by $e^{\alpha t}(\alpha>0)$, we get

$$
\frac{\mathrm{d}}{\mathrm{d} t}\left(e^{\alpha t} E_{n}(t)\right)+e^{\alpha t}\left\|\nabla u_{n t}\right\|^{2}=\alpha e^{\alpha t} E_{n}(t)
$$

and

$$
e^{\alpha t} E_{n}(t)+\int_{0}^{t} e^{\alpha \tau}\left\|\nabla u_{n \tau}\right\|^{2} \mathrm{~d} \tau=E_{n}(0)+\alpha \int_{0}^{t} e^{\alpha \tau} E_{n}(\tau) \mathrm{d} \tau, \quad 0 \leq t<\infty
$$

From $\left(\mathrm{H}_{2}\right)$, Lemma 2.2 and Lemma 4.1, we get

$$
\begin{aligned}
& \sum_{i=1}^{N} \int_{\Omega} G_{i}\left(u_{n x_{i}}\right) \mathrm{d} x-\int_{\Omega} F\left(u_{n}\right) \mathrm{d} x \\
& \quad \leq \frac{a}{2}\left\|\nabla u_{n}\right\|^{2}+\sum_{i=1}^{N} \int_{\Omega} \bar{G}_{i}\left(u_{n x_{i}}\right) \mathrm{d} x-\frac{1}{r+1} \int_{\Omega} u_{n} f\left(u_{n}\right) \mathrm{d} x \\
& \quad \leq \frac{a}{2}\left\|\nabla u_{n}\right\|^{2}+\sum_{i=1}^{N} \int_{\Omega} u_{n x_{i}} \bar{\sigma}_{i}\left(u_{n x_{i}}\right) \mathrm{d} x-\frac{1}{r+1} \int_{\Omega} u_{n} f\left(u_{n}\right) \mathrm{d} x \\
& \quad \leq a\left\|\nabla u_{n}\right\|^{2}+\sum_{i=1}^{N} \int_{\Omega} u_{n x_{i}} \bar{\sigma}_{i}\left(u_{n x_{i}}\right) \mathrm{d} x-\frac{1}{r+1} \int_{\Omega} u_{n} f\left(u_{n}\right) \mathrm{d} x \\
& \quad=\sum_{i=1}^{N} \int_{\Omega} u_{n x_{i}} \sigma_{i}\left(u_{n x_{i}}\right) \mathrm{d} x-\frac{1}{r+1} \int_{\Omega} u_{n} f\left(u_{n}\right) \mathrm{d} x \\
& \quad=\frac{r}{r+1} \sum_{i=1}^{N} \int_{\Omega} u_{n x_{i}} \sigma_{i}\left(u_{n x_{i}}\right) \mathrm{d} x+\frac{1}{r+1} I\left(u_{n}\right) \\
& \quad \leq \frac{1}{1-\delta_{1}} \frac{r}{r+1} I\left(u_{n}\right)+\frac{r}{r+1} I\left(u_{n}\right) \\
& =C\left(r, \delta_{1}\right) I\left(u_{n}\right) \\
& =C\left(r, \delta_{1}\right)\left\|u_{n t}\right\|^{2}-C\left(r, \delta_{1}\right) \frac{\mathrm{d}}{\mathrm{d} t}\left(\left(u_{n t}, u_{n}\right)+\frac{1}{2}\left\|\nabla u_{n}\right\|\right),
\end{aligned}
$$


Xu et al. Boundary Value Problems 2013, 2013:42

Page 10 of 13

where

$$
C\left(r, \delta_{1}\right)=\frac{1}{1-\delta_{1}} \frac{r}{r+1}+\frac{1}{r+1} .
$$

Hence we have

$$
\begin{aligned}
& \int_{0}^{t} e^{\alpha \tau} E_{n}(\tau) \mathrm{d} \tau \\
& =\int_{0}^{t} e^{\alpha \tau}\left(\frac{1}{2}\left\|u_{n \tau}\right\|^{2}+\sum_{i=1}^{N} \int_{\Omega} G_{i}\left(u_{n x_{i}}\right) \mathrm{d} x-\int_{\Omega} F\left(u_{n}\right) \mathrm{d} x\right) \mathrm{d} \tau \\
& \leq\left(\frac{1}{2}+C\left(r, \delta_{1}\right)\right) \int_{0}^{t} e^{\alpha \tau}\left\|u_{n \tau}\right\|^{2} \mathrm{~d} \tau \\
& \quad-C\left(r, \delta_{1}\right) \int_{0}^{t} e^{\alpha \tau} \frac{\mathrm{d}}{\mathrm{d} t}\left(\left(u_{n \tau}, u_{n}\right)+\frac{1}{2}\left\|\nabla u_{n}\right\|^{2}\right) \mathrm{d} \tau
\end{aligned}
$$

and

$$
\begin{aligned}
& -\int_{0}^{t} e^{\alpha \tau} \frac{\mathrm{d}}{\mathrm{d} t}\left(\left(u_{n \tau}, u_{n}\right)+\frac{1}{2}\left\|\nabla u_{n}\right\|^{2}\right) \mathrm{d} \tau \\
& =\left(u_{n t}(0), u_{n}(0)\right)+\frac{1}{2}\left\|\nabla u_{n}(0)\right\|^{2}-e^{\alpha t}\left(\left(u_{n t}, u_{n}\right)+\frac{1}{2}\left\|\nabla u_{n}\right\|^{2}\right) \\
& \quad+\alpha \int_{0}^{t} e^{\alpha \tau}\left(\left(u_{n \tau}, u_{n}\right)+\frac{1}{2}\left\|\nabla u_{n}\right\|^{2}\right) \mathrm{d} \tau \\
& \leq \frac{1}{2}\left(\left\|u_{n t}(0)\right\|^{2}+\left\|u_{n}(0)\right\|^{2}+\left\|\nabla u_{n}(0)\right\|^{2}\right) \\
& \quad+\frac{1}{2} e^{\alpha t}\left(\left\|u_{n t}\right\|^{2}+\left\|u_{n}\right\|^{2}+\left\|\nabla u_{n}\right\|^{2}\right) \\
& \quad+\frac{\alpha}{2} \int_{0}^{t} e^{\alpha \tau}\left(\left\|u_{n \tau}\right\|^{2}+\left\|u_{n}\right\|^{2}+\left\|\nabla u_{n}\right\|^{2}\right) \mathrm{d} \tau .
\end{aligned}
$$

From

$$
\frac{1}{2}\left\|u_{n t}\right\|^{2}+\sum_{i=1}^{N} \int_{\Omega} G_{i}\left(u_{n x_{i}}\right) \mathrm{d} x-\int_{\Omega} F\left(u_{n}\right) \mathrm{d} x=E_{n}(t)
$$

we get

$$
\frac{1}{2}\left\|u_{n t}\right\|^{2}+\frac{1}{l+1} \sum_{i=1}^{N} \int_{\Omega} u_{n x_{i}} \sigma_{i}\left(u_{n x_{i}}\right) \mathrm{d} x-\frac{1}{p+1} \int_{\Omega} u_{n} f\left(u_{n}\right) \mathrm{d} x \leq E_{n}(t)
$$

and

$$
\frac{1}{2}\left\|u_{n t}\right\|^{2}+\frac{p-l}{(p+1)(l+1)} \sum_{i=1}^{N} \int_{\Omega} u_{n x_{i}} \sigma_{i}\left(u_{n x_{i}}\right) \mathrm{d} x+\frac{1}{p+1} I\left(u_{n}\right) \leq E_{n}(t)
$$


which together with $u_{n} \in W$ for sufficiently large $n$ gives

$$
\begin{aligned}
& \frac{1}{2}\left\|u_{n t}\right\|^{2}+\frac{p-l}{(p+1)(l+1)} \sum_{i=1}^{N} \int_{\Omega} u_{n x_{i}} \sigma_{i}\left(u_{n x_{i}}\right) \mathrm{d} x \leq E_{n}(t), \\
& \frac{1}{2}\left\|u_{n t}\right\|^{2}+\frac{p-l}{(p+1)(l+1)}\left(a\left\|\nabla u_{n}\right\|^{2}+\sum_{i=1}^{N} \int_{\Omega} u_{n x_{i}} \bar{\sigma}_{i}\left(u_{n x_{i}}\right) \mathrm{d} x\right) \leq E_{n}(t),
\end{aligned}
$$

and

$$
\frac{1}{2}\left\|u_{n t}\right\|^{2}+\frac{p-l}{(p+1)(l+1)} a\left\|\nabla u_{n}\right\|^{2} \leq E_{n}(t), \quad 0 \leq t<\infty .
$$

From (4.8) and the Poincaré inequality $\|\nabla u\|^{2} \geq \lambda_{1}\|u\|^{2}$, it follows that there exists a constant $C_{0}=C_{0}\left(p, l, a, \lambda_{1}\right)>0$ such that

$$
\left(\left\|u_{n t}\right\|^{2}+\left\|u_{n}\right\|^{2}+\left\|\nabla u_{n}\right\|^{2}\right) \leq C_{0} E_{n}(t), \quad 0 \leq t<\infty .
$$

From (4.5)-(4.10) it follows that there exists a $C_{0}$ such that

$$
\begin{aligned}
e^{\alpha t} E_{n}(t)+\int_{0}^{t} e^{\alpha \tau}\left\|\nabla u_{n \tau}\right\|^{2} \mathrm{~d} \tau \\
\leq\left(C_{0} \alpha+1\right) E_{n}(0)+\left(\frac{1}{2}+C\left(r, \delta_{1}\right)\right) \alpha \int_{0}^{t}\left\|u_{n \tau}\right\|^{2} \mathrm{~d} \tau \\
\quad+\alpha C_{0} e^{\alpha t} E_{n}(t)+\alpha^{2} C_{0} \int_{0}^{t} e^{\alpha \tau} E_{n}(\tau) \mathrm{d} \tau
\end{aligned}
$$

Choose $\alpha$ such that

$$
0<\alpha<\min \left\{\frac{1}{2 C_{0}}, \frac{\lambda_{1}}{\frac{1}{2}+C\left(r, \delta_{1}\right)}\right\} .
$$

Then from (4.11) we get

$$
\begin{aligned}
e^{\alpha t} E_{n}(t) & \leq 2\left(C_{0} \alpha+1\right) E_{n}(0)+2 \alpha^{2} C_{0} \int_{0}^{t} e^{\alpha \tau} E_{n}(\tau) \mathrm{d} \tau \\
& \leq 2\left(C_{0} \alpha+1\right) d_{0}+2 \alpha^{2} C_{0} \int_{0}^{t} e^{\alpha \tau} E_{n}(\tau) \mathrm{d} \tau \\
& \leq 3 d_{0}+2 \alpha C_{0} \int_{0}^{t} e^{\alpha \tau} E_{n}(\tau) \mathrm{d} \tau .
\end{aligned}
$$

From this and the Gronwall inequality, we get

$$
e^{\alpha t} E_{n}(t) \leq 3 d_{0} e^{-2 \alpha^{2} C_{0} t}
$$

and

$$
E_{n}(t) \leq 3 d_{0} e^{-\lambda t}, \quad 0 \leq t<\infty, \quad \lambda=\alpha\left(1-2 C_{0} \alpha\right)>0 .
$$


On the other hand, from (4.8) we get

$$
\frac{1}{2}\left\|u_{n t}\right\|^{2}+\frac{p-l}{(p+1)(l+1)} A\left\|\nabla u_{n}\right\|_{m+1}^{m+1} \leq E_{n}(t), \quad 0 \leq t<\infty .
$$

Hence, there exists a $C_{1}=C_{1}(p, l, A)$ such that

$$
\left\|u_{n t}\right\|^{2}+\left\|\nabla u_{n}\right\|_{m+1}^{m+1} \leq C_{1} E_{n}(t), \quad 0 \leq t<\infty .
$$

Let $\left\{u_{v}\right\}$ be the subsequence of $\left\{u_{n}\right\}$ in the proof of Theorem 3.2. Then from (4.13) and(4.12), we obtain

$$
\begin{aligned}
& \left\|u_{t}\right\|^{2}+\|\nabla u\|_{m+1}^{m+1} \\
& \quad \leq \liminf _{v \rightarrow \infty}\left\|u_{v t}\right\|^{2}+\liminf _{v \rightarrow \infty}\left\|\nabla u_{v}\right\|_{m+1}^{m+1} \\
& \quad \leq \liminf _{v \rightarrow \infty}\left(\left\|u_{v t}\right\|^{2}+\left\|\nabla u_{v}\right\|_{m+1}^{m+1}\right) \\
& \quad \leq \liminf _{v \rightarrow \infty} C_{1} E_{v}(t) \leq 3 d_{0} C_{1} e^{-\lambda t}, \quad 0 \leq t<\infty,
\end{aligned}
$$

which gives (4.4), where $C=3 d_{0} C_{1}, \lambda=\alpha\left(1-2 C_{0} \alpha\right)$.

\section{Competing interests}

The authors declare that they have no competing interests.

\section{Authors' contributions}

The work presented here was carried out in collaboration between all authors. RZ organised this paper and found the topic of this paper. He introduced this problem and suggested the methods and the outline of the proofs. JL finished the proof of the global existence and YN finished the long time behaviour part. SC discussed all the problems arising in the research and provided many good ideas for proving the problems. All authors have contributed to, seen and approved the manuscript.

\section{Author details}

${ }^{1}$ College of Science, Harbin Engineering University, Harbin, 150001, People's Republic of China. ${ }^{2}$ College of Automation, Harbin Engineering University, Harbin, 150001, People's Republic of China. ${ }^{3}$ Department of Mathematics, Cape Breton University, Sydney, B1P 6L2, Canada.

\section{Acknowledgements}

We thank the referees for their valuable suggestions which helped us improve the paper so much. This work was supported by the National Natural Science Foundation of China (11101102), Ph.D. Programs Foundation of the Ministry of Education of China (20102304120022), the Support Plan for the Young College Academic Backbone of Heilongjiang Province (1252G020), the Natural Science Foundation of Heilongjiang Province (A201014), Science and Technology Research Project of the Department of Education of Heilongjiang Province (12521401), Foundational Science Foundation of Harbin Engineering University and Fundamental Research Funds for the Central Universities.

Received: 24 April 2012 Accepted: 11 February 2013 Published: 1 March 2013

\section{References}

1. Greenberg, JM: On the existence, uniqueness and stability of solutions of the equation $\rho_{0} X_{x x}=E\left(X_{x}\right) X_{x x}+\lambda X_{x x t}$. J. Math. Anal. Appl. 25, 575-591 (1969)

2. Greenberg, JM, MacCamy, RC, Mizel, JJ: On the existence, uniqueness and stability of solutions of the equation $\rho\left(u_{x}\right) u_{x x}+\lambda u_{x x t}=\rho_{0} u_{t t} . J$. Math. Mech. 17, 707-728 (1968)

3. Andrews, G: On the existence of solutions to the equation $u_{t t}-u_{x x t}=\sigma\left(u_{x}\right)_{x}$. J. Differ. Equ. 35, 200-231 (1980)

4. Andrews, G, Ball, JM: Asymptotic behavior and changes in phase in one-dimensional nonlinear viscoelasticity J. Differ. Equ. 44, 306-341 (1982)

5. Dafermos, CM: The mixed initial-boundary value problem for the equations of nonlinear one-dimensional visco-elasticity. J. Differ. Equ. 6, 71-86 (1969)

6. Davis, P: A quasi-linear hyperbolic and related third-order equation. J. Math. Anal. Appl. 51, 596-606 (1975)

7. Engler, H: Strong solutions for strongly damped quasilinear wave equations. Contemp. Math. 64, 219-237 (1987)

8. Greenberg, JM, MacCamy, RC: On the exponential stability of solutions of $E\left(u_{x}\right) u_{x x}+\lambda u_{x t x}=\rho u_{t t}$. J. Math. Anal. Appl. $31,406-417(1970)$ 
9. Liu, Y, Liu, D: Initial boundary value problem, periodic boundary problem and initial value problem of equation $u_{t t}=u_{x x t}+\sigma\left(u_{x}\right)_{x}$. Chin. Ann. Math., Ser. A 9, 459-470 (1988) (in Chinese)

10. Yamada, Y: Some remarks on the equation $y_{t t}-\sigma\left(y_{x}\right) y_{x x}-y_{x t x}=f$. Osaka J. Math. 17, 303-323 (1980)

11. Yang, Z, Song, C: Blow-up of solutions for a class of quasilinear evolution equations. Nonlinear Anal., Theory Methods Appl. 28, 2017-2032 (1997)

12. Ang, DD, Dinh, PN: Strong solutions of a quasilinear wave, equation with nonlinear damping. SIAM J. Math. Anal. 102 337-347 (1988)

13. Clements, J: Existence theorems for a quasilinear evolution equation. SIAM J. Appl. Math. 226, 745-752 (1974)

14. Clements, J: On the existence and uniqueness of solutions of the equation $u_{t t}-\partial \sigma_{i}\left(u_{x_{j}}\right) / \partial x_{i}-D_{N} u_{t}=f$. Can. Math. Bull. 18, 181-187 (1975)

15. Yang, Z: Existence and asymptotic behaviour of solutions for a class of quasilinear evolution equations with nonlinear damping and source terms. Math. Methods Appl. Sci. 25, 795-814 (2002)

16. Liu, Y, Zhao, J: Multidimensional viscoelasticity equations with nonlinear damping and source terms. Nonlinear Anal. $56,851-865$ (2004)

17. Xu, R: Asymptotic behavior and blow-up of solutions for the viscous diffusion equation. Appl. Math. Lett. 20, 255-259 (2007)

18. $\mathrm{Xu}, \mathrm{R}, \mathrm{Zhao}, \mathrm{X}$, Shen, J: Asymptotic behaviour of solution for fourth order wave equation with dispersive and dissipative terms. Appl. Math. Mech. 29, 259-262 (2008)

19. Liu, Y, Xu, R, Yu, T: Global existence, nonexistence and asymptotic behavior of solutions for the Cauchy problem of semilinear heat equations. Nonlinear Anal. 68, 3332-3348 (2008)

20. $\mathrm{Xu}, \mathrm{R}, \mathrm{Liu}, \mathrm{Y}$ : Asymptotic behavior of solutions for initial-boundary value problems for strongly damped nonlinear wave equations. Nonlinear Anal. 69, 2492-2495 (2008)

21. Xu, R: Global existence, blow up and asymptotic behaviour of solutions for nonlinear Klein-Gordon equation with dissipative term. Math. Methods Appl. Sci. 33, 831-844 (2010)

22. Jiang, $X, X u, R$ : Global well-posedness for semilinear hyperbolic equations with dissipative term. J. Appl. Math. Comput. 38, 467-487 (2012)

doi:10.1186/1687-2770-2013-42

Cite this article as: Xu et al.: Asymptotic behaviour of solution for multidimensional viscoelasticity equation with nonlinear source term. Boundary Value Problems 2013 2013:42.

\section{Submit your manuscript to a SpringerOpen ${ }^{\odot}$ journal and benefit from:}

- Convenient online submission

- Rigorous peer review

- Immediate publication on acceptance

- Open access: articles freely available online

- High visibility within the field

- Retaining the copyright to your article 